\title{
The Use of Single Lenses in the Radio Frequency Mass Spectrometers
}

${ }^{1}$ T. Daumenov, ${ }^{2}$ M. A. Khizirova, ${ }^{3}$ A. S. Baikenov, ${ }^{4}$ A. O. Kassimov, ${ }^{5}$ S. T. Amanzholova

${ }_{1,2,3,4}$ Department of Technical Physics, Almaty University of Power Engineering and Telecommunications

(AUPET), Bytesunov St 126/1, Almaty, Kazakhstan

${ }^{5}$ Department of Computer Engineering and Telecommunications, International IT University, Rozybakiyev st. 94-51

Almaty, Kazakhstan

Email:dautl@mail.ru,hizirova73@mail.ru,baikenoff@yandex.kz,64razak@mail.ru,shokatarva@gmail.com

Received: $16^{\text {th }}$ August 2018, Accepted: $19^{\text {th }}$ September 2018, Published: $31^{\text {st }}$ October 2018

\begin{abstract}
The possibility of using a single lens to increase the resolution of the radio-frequency mass spectrometer is shown. The problem is solved by calculating the additional energy of ions when they pass through the electrostatic field of a single lens with rotational symmetry.
\end{abstract}

Keywords

Single Lens Selecting Cascade, Radio-Frequency Mass Spectrometer, An Increase of Energy Resolution, Ion Flux

\section{Introduction}

Radio-frequency mass spectrometers (RFMS) [1] are known, which are used to analyze the ion and molecular composition of the upper atmosphere (figure 1), to measure partial pressures in vacuum technology. A typical node of these devices is a selection cascade, consisting, as a rule, of three plane-parallel grids $\left(C_{2}, C_{3}, C_{4}\right)$, located at an equal distance s from each other, which are fed negative accelerating voltage $U$, and the middle grid $\left(C_{3}\right)$ and high-frequency sinusoidal voltage with amplitude. Between the cathode $\mathrm{K}$ and a grid accelerates ions of the applied voltage.

While passing the ion beam through selecting cascade, depending on the speed of the ions change their energy. Indeed, if the ions are accelerated when moving in the region between the second and third grids, and after passing through the third grid, its potential will change so that the ions between the third and fourth grids will also accelerate, then they will receive additional energy at the output of the system. The problem is that at a given accelerating voltage $V_{0}$ on the grids and the frequency of alternating voltage $\omega$, the ions of a certain mass $m$ and charge $q$ passing through the selection stage, acquire the greatest increase in energy $\Delta W$. In order for the ions to get the maximum energy, it is necessary that their time of flight is quite certain and the phase of flight to the area between the second and third grids is optimal.

With the same ion energy, the first condition is satisfied only for ions with a certain mass (velocity). Feeding on an additional fifth grid retarding potential $\left(V_{0}+V_{T}\right)$, a slightly larger accelerating, which secrete ions that have received additional energy, i.e. having a well-defined mass.

The energy increment of the ions $\Delta W$ on the path in the work $C_{2}-C_{4}[1]$ is found assuming that there are no volume charges and that the time of flight of the path ions $C_{2}-C_{4}$ does not depend on the small amplitude of the alternating field on the third grid:

$\Delta W=\frac{q U_{m} v}{s \omega}\left[\cos \theta-2 \cos \left(\frac{\omega s}{v}+\theta\right)+\cos \left(\frac{2 \omega s}{v}+\theta\right)\right]$

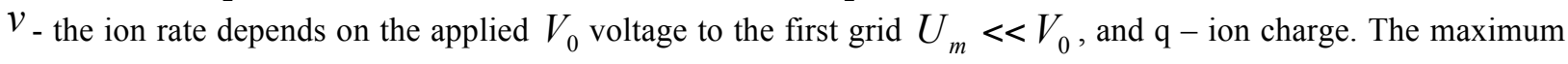
value of the energy when the initial phase $\theta$ changes meets the condition:

$$
\frac{s \omega}{v}+\theta=\pi
$$

In variation of frequency of maximum energy yields the value of $\theta=46^{0} 26^{\prime}$, herewith $\frac{s \omega}{v}=133^{0} 34^{\prime}$.

From these expressions the ion mass can be calculated as $m=0.266 V_{0} / s^{2} f^{2}$ coal. ed., 


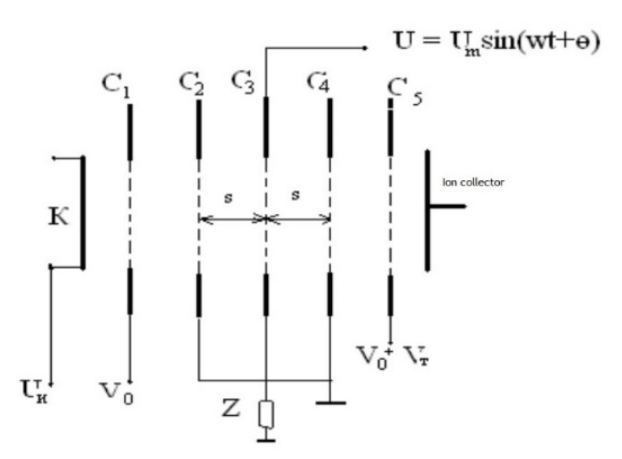

Figure 1: Diagram of Single-Stage RFMS

where $V_{0}$ - accelerating voltage, B; $\mathrm{f}-$ frequency, $\mathrm{MHz} ; \mathrm{s}$ - the distance between the grids, sM.

\section{Disadvantages of the Classical Scheme of RFMS}

The resolution of the considered radio-frequency mass spectrometric lamp is extremely small and is several units (34). Cascading of analyzers is used to increase the resolution.

The resolution can be increased by increasing the number of accelerating sections in one cascade, as well as the number of cascades. At the same time, the ion flight time from one stage to another must be a multiple of the period of the supply voltage.

In multistage analyzers, the sensitivity of the mass spectrometer can be significantly reduced due to the low transparency of the grids. For example, when used in a three-stage device with one section in each cascade of grids with transparency $80 \%$ sensitivity is reduced by an order of magnitude.

To study the composition of neutral and ionized gases of the upper atmosphere in the USSR developed RFMS: MH6401, MH6403, MH6405, MH6407 [2]. The devices are small in size, weight and power consumption.

The presence of grid electrodes in the RFMS significantly affects the sensitivity of the device. First, the meshes mechanically cover a part of the space for the ions to pass through. Secondly, the structure of equipotential surfaces in the area occupied by grids is cellular, which causes the scattering of ions.

In addition, during operation under the action of ions on the grids can form a bad conductive film, which over time can lead to uncontrolled changes in the mode of operation of the device, in particular, to the loss of its sensitivity and resolution. These unwanted effects are compounded when used in RCMS several selecting cascades. The paper discusses ways to address these shortcomings.

\section{Brushless RFMS Analyzer with Piecewise Linear Potential Distribution on the System Axis}

It discusses the question of the possibility of eliminating the above drawbacks analyzer ROMS by replacing the grid selecting cascades of single lenses with large focal lengths. Suppose that single lenses consist of three diaphragms with diameters of holes $\mathrm{d}$, which are at equal distance $\mathrm{s}$ from each other.

Let the potentials of the extreme electrodes $-U_{1}$, and the average $-U_{2}$. The origin of the coordinate system is compatible with the left extreme electrode $(\mathrm{z}=0)$.

Suppose that there are no volume charges and the time of flight of ions between the electrodes does not depend on the small amplitude of the alternating voltage $U_{m}$ with the initial phase $\psi$ applied to the middle electrode relative to the extreme electrodes:

$$
u=U_{m} e^{j(\omega t+\psi)}
$$

Potential distribution on the axis of the system can be represented by a piecewise linear function:

$$
\Phi(z)=\left\{\begin{array}{lc}
U_{1}+\Delta U \frac{z}{s}, & 0 \leq z \leq s, \\
U_{2}+\Delta U \frac{(s-z)}{s}, & s \leq z \leq 2 s
\end{array}\right\}
$$

where

$$
\Delta U=U_{2}-U_{1}
$$

is a small value.

Then the ion velocity in the intervals between the electrodes can be represented as: 


$$
\begin{aligned}
& v_{1}=\sqrt{-\frac{2 e}{m}\left(U_{1}+\frac{\Delta U}{s} z\right), \quad 0 \leq z \leq s,} \\
& v_{2}=\sqrt{-\frac{2 e}{m}\left(U_{2}+\frac{\Delta U}{s}(s-z)\right)}, \quad s \leq z \leq 2 s
\end{aligned}
$$

The energy increment $\Delta W$ of ions passing through the system is determined as follows:

$$
\Delta W=\int\left(q U_{m} / s\right) v e^{j(\omega t+\psi)} d t
$$

here, the value of $\mathrm{q}$ is the linear charge of ions. When applying the formula (6), note that when the ion reaches a certain mass $m$ of the plane $\mathrm{z}=\mathrm{s}$, the phase of the high-frequency voltage should change to $\pi$ :

$$
\Delta W=\int_{0}^{s / v_{1}}\left(q U_{m} / s\right) v_{1} e^{j(\omega t+\psi)} d t-\int_{s / v_{2}}^{2 s / v_{1}}\left(q U_{m} / s\right) v_{2} e^{j(\omega t+\psi)} d t
$$

Next, we note that the values of q and $U_{m}$ can be taken out of the integral, in addition, using a small value $\Delta U$, using the appropriate expansion and neglecting small values of high orders, rewrite the expression (7) as follows:

$$
\Delta W=\left(q U_{m} / s\right) \sqrt{-\frac{2 e}{m} U_{1}}\left(1+\frac{\Delta U}{U_{1}} \frac{z}{s}\right)\left[\int_{0}^{s / v_{1}} e^{j(\omega t+\psi)} d t-\sqrt{\frac{U_{2}}{U_{1}}}\left(1+\frac{\Delta U}{2 U_{2}}\right)_{s / v_{2}}^{2 s / v_{1}} e^{j(\omega t+\psi)}\right]
$$

In the expression (8) we used the value of the average speed on the field interval. After the integration of expression (8), the energy increment will take the form:

$$
\Delta W=\left(q U_{m} / s\right) \sqrt{-\frac{2 e}{m} U_{1}}\left(1+\frac{\Delta U}{2 U_{1} s} \frac{z}{s}\right)\left[e^{j \psi}-e^{j\left(\frac{\omega s}{v_{1}}+\psi\right)}-\sqrt{\frac{U_{2}}{U_{1}}}\left(1+\frac{\Delta U}{U_{1}}\right)\left(e^{j\left(\frac{\omega s}{v_{2}}+\psi\right)}-e^{j\left(\frac{\omega s}{v_{1}}+\psi\right)}\right)\right]
$$

The maximum value of the expression enclosed in a square bracket is obtained when the conditions are met

$$
\frac{\omega S}{v_{i}}+\psi=\pi, \quad(\mathrm{i}=1,2)
$$

which in view of the approximate equality $v_{1} \approx v_{2}$ can be agreed between them, indeed, the difference of the initial phases does not exceed the value $\frac{\Delta \psi}{\psi} \approx \frac{\Delta v}{v_{i}}$.

For the maximum value of the energy $\Delta W_{\max }$ increment we obtain

$$
\Delta W_{\max }=\frac{q U_{m}}{\omega s} \sqrt{-\frac{2 e}{m} U_{1}}\left(1+\frac{\Delta U}{2 U_{1}} \frac{z}{s}\right)\left[1+e^{j \psi}+\sqrt{\frac{U_{2}}{U_{1}}}\left(1+\frac{\Delta U}{2 U_{2}}\right)\left(1+e^{j\left(\frac{\omega s}{v_{2}}+\psi\right)}\right)\right]
$$

In the special case, when the maximum value $U_{1}=U_{2}(\Delta U=0)$ of the energy increment takes the form:

$$
\Delta W_{\max }=\frac{q U_{m} v}{\omega s}\left[e^{j \psi}+e^{j\left(\frac{2 \omega s}{v_{1}}+\psi\right)}+2\right]
$$

what is consistent with the known results [1].

Thus, if we assume that the transparency coefficient of each grid of the selecting cascade is 0.9 , then about $30 \%$ of ions are lost when passing through one cascade. The use of a single lens virtually eliminates the loss of ions in the RFMS analyzers, thereby significantly increasing the sensitivity of the device. From comparisons of the last two expressions shows that the $\Delta U>0$ maximum energy gain of ions is obtained when using the single lenses in the accelerating - decelerating mode, i.e. there is a real possibility of improving resolution RCMS.

\section{Brushless RFMS Analyzer with Arbitrary Potential Distribution on the System Axis}

The focusing properties of single lenses with constant potentials on the electrodes creating an electrostatic field in the lens space are well known. 
This subsection discusses the influence of the electric fields of single lenses on ion flows, if the electrodes of the lenses in addition to the constant potentials are also variable in time potentials, creating modulating electric fields. For simplicity of consideration it is accepted that the direction of the initial velocity of charged particles coincides with the direction of electric field lines of alternating field.

Consider a three-electrode single lens, the electrodes of which consist of three flat coaxial diaphragms with circular holes of the same diameter, arranged in parallel with each other $S$ and at an equal distance from each other (figure $2)$.

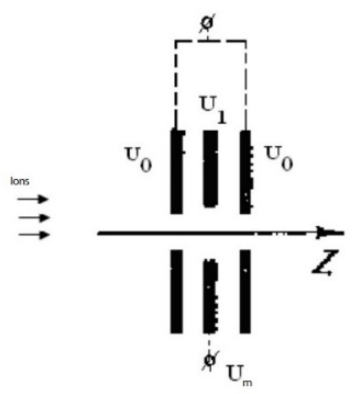

Figure 2: Connecting a High-Frequency Field to the Electrodes of a Single Lens for Mass Analysis of Ion Flows

The origin of the coordinate system is compatible with the plane of the extreme left aperture, let the $z$ axis be the axis of symmetry. The axial distribution of the electrostatic potential $\Phi(z)$ is presented as:

$$
\Phi(z)=\Phi_{0}\left(1-\frac{\chi^{2}}{1+\left(\frac{z-s}{d}\right)^{2}}\right)
$$

- free space potential, $\chi^{2}$-a numerical parameter varying within $0<\chi^{2}<1, d$-the diameter of the orifices of the diaphragms.

First, we consider the case when a single lens passes a beam of ions of different masses. Let the average electrode of the lens relative to the extreme voltage is applied with frequency $\mathcal{W}$, amplitude $U_{m}$ and initial phase $\psi_{0}$ :

$$
u=U_{m} \sin \left(w t+\psi_{0}\right)
$$

In this case, the distance $S$ between the flat electrodes and the frequency $w$ of the alternating voltage are selected so that the change of the voltage phase $\pi$ at an angle occurs at the time of passage of the ion of a certain mass $m$ of the plane of the middle electrode:

$$
u=-U_{m} \sin \left(w t^{*}+\psi_{0}\right)
$$

$t \quad-$ the time of crossing the plane of the left electrode of the lens, $t^{*}$ - the moment of the ion passing through the plane of the middle electrode.

The additional energy $\Delta W$ gained by the ion considered when passing it through a single lens can be represented by the expression:

$$
\begin{aligned}
\Delta W= & \frac{q U_{m}}{\Phi_{0}} \frac{1}{s}\left[\int_{0}^{s} \Phi(z) \sin \left(\frac{w z}{v_{0}}+\psi_{0}\right) d z-\int_{s}^{2 s} \Phi(z) \sin \left(\frac{w z}{v_{0}}+\psi_{0}\right) d z\right]+ \\
& \frac{q U_{m}}{\Phi_{0}}\left[\int_{0}^{s} \Phi^{\prime}(z) \sin \left(\frac{w z}{v_{0}}+\psi_{0}\right) d z-\int_{s}^{2 s} \Phi^{\prime}(z) \sin \left(\frac{w z}{v_{0}}+\psi_{0}\right) d z\right]
\end{aligned}
$$

$q$ - charge, $v_{0}$ - ion velocity determined by the value of the accelerating potential

$v_{0}=\sqrt{\frac{2 q}{m} \Phi_{0}}$,

moreover, the amplitude of the alternating voltage satisfies the condition $U_{m}<<\Phi_{0}$. The strokes denote differentiation in the coordinate $\Delta W$. After certain transformations, additional energy takes the form: 
$\Delta W=\Delta W_{0}+\Delta W_{\pi}$

where

$$
\begin{aligned}
& \Delta W_{0}=\frac{q U_{m} v_{0}}{w s} f_{1}\left(\psi_{0}\right) ; \\
& \Delta W_{\pi}=q U_{m} \chi^{2} f_{2}\left(\psi_{0}\right) ;
\end{aligned}
$$

Here are the symbols

$$
f_{1}\left(\psi_{0}\right)=\cos \psi_{0}-2 \cos \left(\frac{w s}{v_{0}}+\psi_{0}\right)+\cos \left(2 \frac{w s}{v_{0}}+\psi_{0}\right)
$$

First, we consider the case when a single lens passes a beam of ions of different masses. Let the medium electrode of the lens relative to the extreme voltage is applied with frequency $w$, amplitude $U_{m}$ and initial phase $\psi_{0}$.

$$
\begin{aligned}
f_{2}\left(\psi_{0}\right)= & {\left[2 \cos \frac{w s}{v_{0}}\left(\frac{1}{1+\left(\frac{s}{d}\right)^{2}} \cos \frac{w s}{v_{0}}-1\right)+\frac{1}{s}\left(I_{4}-I_{3}\right)+\frac{w}{v_{0}}\left(I_{2}-I_{1}\right)\right] \sin \psi_{0}+} \\
& {\left[2 \sin \frac{w s}{v_{0}}\left(\cos \frac{w s}{v_{0}}-\frac{1}{1+\left(\frac{s}{d}\right)^{2}}\right)+\frac{1}{s}\left(I_{2}-I_{1}\right)-\frac{w}{v_{0}}\left(I_{4}-I_{3}\right)\right] \cos \psi_{0} }
\end{aligned}
$$

where

$$
\begin{array}{ll}
I_{1}=\int_{0}^{s} f(z) \sin \frac{w z}{v_{0}} d z, & I_{2}=\int_{s}^{2 s} f(z) \sin \frac{w z}{v_{0}} d z, \\
I_{3}=\int_{0}^{s} f(z) \cos \frac{w z}{v_{0}} d z, & I_{4}=\int_{s}^{2 s} f(z) \cos \frac{w z}{v_{0}} d z, \\
f(z)=\frac{1}{\left[1+\left(\frac{z-s}{d}\right)^{2}\right]} . &
\end{array}
$$

$\Delta W_{0}$ - the part of the additional energy that the ions acquire in the absence of an inhomogeneous electric field, which coincides with the formula obtained in [1]. Another part of the additional energy $\Delta W_{n}$ is due to the action of a single lens. The graphs (figure 3) show the dependence of the value $\Delta W / q m$ of the initial phase of alternating voltage.

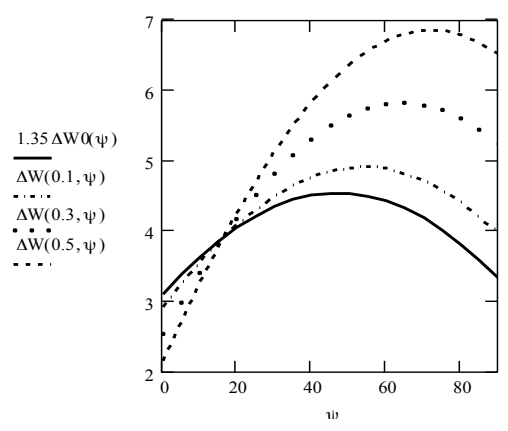


Figure 3: Dependence of the Value $\frac{\Delta W}{q m}(\psi)$ for Different $\chi^{2}-0,1 ; 0,2 ; 0,3$

The dependence of the additional energy acquired by ions in the absence of a non-uniform electric field is shown separately. As you can see, the maximum value $f\left(\psi_{0}\right)$ of the function is achieved when this result coincides $\psi_{0}=46^{0}$ with the data of [1].

The same graph shows the total effect at different values $\chi^{2}=0.1 ; 0.3 ; 0.5$;

With the increase of this value, i.e. with the increase in the optical power of a single lens, there is an increase in the additional energy of ions. This effect can be used to create a lens radio frequency mass analyzer.

In particular, as follows from the graph, when $\chi^{2}=0.3$; increasing the resolution of one selection of the RFMS cascade in the mass analysis of different ions is $22.2 \%$.

\section{Conclusion}

Thus, the disadvantage of the analyzer of the radio-frequency mass spectrometer of the three-grid selection stage is the fact that the grids are a mechanical obstacle in the way of ions [3,4,5]. In a single-stage mass spectrometer analyzer, ions pass through the grids at least three times, and even for grids with very good transparency, the total transparency of the cascade decreases to $50 \%$. Near the grid cells, there is also a sagging of equipotential surfaces, which have a scattering effect on the ions. These factors affect the sensitivity of the device, in the end, and its resolution. In addition, during the operation of the grid can be contaminated and covered with a thin film, which will lead to uncontrolled operation of the mass spectrometer.

Figure 4 shows a block diagram of a single-stage RFMS with an analyzing cascade based on a single lens.

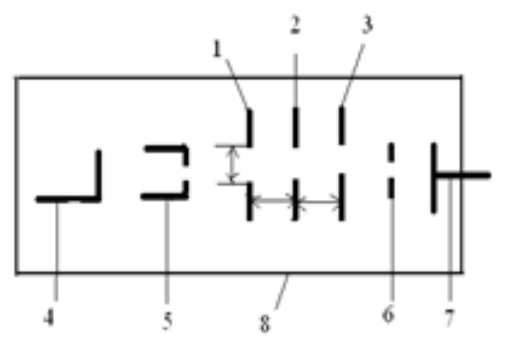

1, 2, 3- single lens, 4- cathode, 5- ion source, 6- holding electrode, 7- ion collector, 8- corpus

Figure 4: Block Diagram of a Single-Stage RFMS with a Single Lens Analyzer

The device works as follows. The analyzer is a single lens decreases the beam of the analyzed mass.

In the analyzer, the ions receive, depending on their mass, a larger or smaller increase in energy. At a fixed frequency of voltage and energy of ions, only ions with certain mass-to-charge ratios have such a speed that, moving between the first and second diaphragms in the half-cycle, when the field between them is accelerating for the ions, they cross the second diaphragm at the time of the field sign change and pass between the second and third diaphragms also in the accelerating field.

Thus, they receive the maximum increase in energy and fall on the collector. Ions of other masses, passing a single lens, or are inhibited by the field, i.e. lose energy, or receive an insufficient increase in energy and are discarded at the end of the path by the braking potential.

\section{References}

1. Rick G. R. Radio-frequency mass spectrometer//UFN. - 1951. - T. 43. - Page 301-307.

2. Rafalson A. E, Shereshevsky A.M. Mass and spectrometer devices. - M, 1968. - 204 pages.

3. Daumenov T. D., Hizirova M.A., Yakushev E.M. Features of focusing of bunches of charged particles in the presence of fields with periodic modulation//Materials the 4th Mezhd. Conf.: "Nuclear and radiation physics". Almaty, 2003. - Page 259-264.

4. Daumenov T. D. Features of focusing of bunches of charged particles in the presence of fields with periodic modulation.//Uzbek Journal of Physics.-2009. - T.11.-№ 1. - Page 47-51

5. Daumenov T. D. Radio-frequency mass spectrometer.//RK No. 22947 patent 